\title{
Adhesion molecule expression in the lung: a comparison between normal and diffuse interstitial lung disease
}

\author{
A.M. Southcott*, I. Hemingway+, S. Lorimers, K. Sugars", P.G. Hellewell; C.M. Black ${ }^{\circ}$, \\ P.K. Jeffery», A.J.H. Gearing+, D.O. Haskard", R.M. du Bois*
}

\begin{abstract}
Adhesion molecule expression in the lung: a comparison between normal and diffuse interstitial lung disease. A.M. Southcott, I. Hemingway, S. Lorimer, K. Sugars, P.G. Hellewell, C.M. Black, P.K. Jeffery, A.J.H. Gearing, D.O. Haskard, R.M. du Bois. OERS Journals Ltd 1998.

ABSTRACT: Cellular adhesion molecules are crucial determinants of the migration of immune effector cells to the tissues. In chronic inflammatory diseases, upregulation of the expression of these molecules may contribute to the persistent inflammatory process. The aim of this study was to determine whether there is evidence of adhesion molecule expression in chronically inflamed lung.

Soluble adhesion molecules in bronchoalveolar lavage fluid (BALF) were measured by enzyme-linked immunoassay in 54 patients with chronic interstitial lung diseases and 16 normal controls. Adhesion molecule expression in fibrosing alveolitis (FA) lung and in control lung was assessed using immunohistology and reverse transcription-polymerase chain reaction (RT-PCR) amplification.

Soluble intercellular adhesion molecule-1 (ICAM-1) was detected in all but two subjects. There was no difference in ICAM-1 concentration between disease groups and normal subjects. In contrast, soluble E-selectin was detected in 17 of the 70 subjects and was significantly associated with the presence of lung disease $(p=0.0173)$. Furthermore, the presence of soluble E-selectin was associated with a raised lymphocyte percentage in BALF $(p=0.0069)$. Soluble VCAM was only detected in five of the 70 subjects (two normals, three patients). There was no difference in adhesion molecule expression in lung parenchyma between FA and controls assessed by immunohistology and RT-PCR.

The most striking finding of our study was the universal expression of intercellular adhesion molecule-1 in both normal and diseased lung, emphasizing the important role of the lung in immune function. Upregulation of E-selectin may contribute to inflammatory cell accumulation in chronic interstitial lung diseases.

Eur Respir J 1998; 11: 000-000.
\end{abstract}

*Interstitial Lung Disease Unit, Royal Brompton Hospital, London, UK. ${ }^{+}$British BioTechnology Limited, Oxford, UK. \#Dept of Medicine, Royal Postgraduate Medical School, London, UK. 'Dept of Rheumatology, Royal Free Hospital, London, UK. Depts of \$Lung Pathology, and Clinical Pharmacology, National Heart and Lung Institute, London, UK

Correspondence: R.M. du Bois

Royal Brompton Hospital

Sydney Street

London SW3 6NP

UK

Fax: 00441713518435

Keywords: Adhesion molecules cell adhesion

interstitial lung disease

Received: July 171996

Accepted after revision April 161997

This study was supported by the Raynauds and Scleroderma Association, UK.
Diffuse lung diseases such as sarcoidosis and fibrosing alveolitis are characterized by the accumulation at disease sites of large numbers of acute and chronic inflammatory cells and these cells are known to play a significant role in the pathogenesis of the disease process [1]. However, remarkably little is known about how these cells are attracted into the local microenvironment. Control of inflammation is now known to involve a number of membrane adhesion receptors, which may be classified into several families, or superfamilies [2], including the selectins, of which E-selectin (CD62E) is an example, and the immunoglobulin supergene family (IGSF). Examples of IGSF members include intercellular adhesion molecule -1 (ICAM-1) (CD54), vascular cell adhesion molecule 1 (VCAM-1)(CD106) and platelet/endothelial cell adhesion molecule (PECAM) (CD31) (also known as endoCAM). Selectins are believed to mediate the initial rolling of leucocytes along the cell surface and IGSF molecules are essential for the subsequent firm attachment of leucocytes to, and migration through, the cell membrane [3]. In chronic inflammatory disease states, the persistent accumulation and local activation of these inflammatory cells is believed to add to the burden of injury. Upregulation of adhesion molecule expression may, thus, contribute to the persistence of inflammation $[4,5]$.

It is now recognized that cellular adhesion molecules may be found in different forms, either associated with the cell surface or as soluble isoforms within the circulation [6-8]. Soluble forms of adhesion molecules may be produced by cleavage of the molecule or by alternative splicing of messenger ribonucleic acid (mRNA). Their presence, particularly those molecules that are only upregulated in response to triggering by proinflammatory cytokines (Eselectin; VCAM-1) may be used as a marker of active disease. Whether or not these truncated forms are functional is uncertain: they could compete with cell bound ligands and inhibit cell-cell adhesion or, alternatively, initiate a response in a ligand-bearing cell.

This study of the expression of adhesion cell molecules in diffuse lung disease, therefore, had two main objectives. The first was to identify soluble adhesion molecules in the lower respiratory tract using bronchoalveolar lavage (BAL) and, if they were present, to relate them to the type of cell present on the epithelial lining surface. The second 
aim was to characterize the expression of cell surface adhesion molecules in both normal and chronically inflamed lung in fibrosing alveolitis (FA). Although there have been a number of studies of adhesion molecule expression in pulmonary airway disease, both in animal models [9] and in investigations of asthma in humans [10], conclusions have been conflicting. Very few studies have explored the role of adhesion molecules in interstitial lung disease [11]. To examine adhesion molecule expression in the lung parenchyma we used the techniques of immunohistology and reverse transcription-polymerase chain reaction (RT-PCR) to identify the transcription and translation products of the genes that encode ICAM-1 (CD54), VCAM-1 (CD106), and E-selectin (CD29E) and, furthermore, to compare the expression of these molecules in fibrosing lung disease with the normal lung, which is now recognized to be an immunologically active organ even in the absence of disease.

\section{Materials and methods}

Measurement of soluble adhesion molecules in bronchoalveolar lavage fluid

Patients. Fifty four consecutive patients who attended the Interstitial Lung Disease Unit at the Royal Brompton Hospital between July 30, 1990 and July 1, 1991 who required bronchoalveolar lavage (BAL) as part of clinical evaluation (49 at presentation; five who had BAL for the first time during follow-up) were studied.

The group comprised 17 patients with sarcoidosis, 14 with fibrosing alveolitis (FA) (10 with cryptogenic FA (CFA) and four with FA occurring in the context of a rheumatic disease other than systemic sclerosis (SSc)), 20 patients with SSc and three patients with other interstitial lung disease (ILD). The characteristics of these patients are presented in table 1 . The 20 patients with SSc included three with no evidence of FA on computed tomography (CT), all of whom had normal lung volumes and transfer factor of the lung for carbon monoxide $(T \mathrm{~L}, \mathrm{CO})$ values of 76,78 and $89 \%$ predicted. Of the three patients with other ILD, one had hypersensitivity pneumonitis, one had previously diagnosed sarcoidosis with new features suggestive of hypersensitivity pneumonitis and one had pleural and pulmonary fibrosis with previous occupational exposure to asbestosis. Diagnoses were based on typical clinical fea-

Table 1. - Patient characteristics

\begin{tabular}{|c|c|c|c|c|c|c|}
\hline & \multicolumn{2}{|c|}{ Patients } & \multirow[t]{2}{*}{ Smokers $^{+}$} & \multicolumn{3}{|c|}{ Treatment } \\
\hline & $\mathrm{n}$ & $\mathrm{M} / \mathrm{F}$ & & $\begin{array}{l}\text { Pred } \\
\text { only } \\
n\end{array}$ & $\begin{array}{l}\text { Pred } \\
+\underset{n}{\text { Im }}\end{array}$ & $\begin{array}{c}\text { Im } \\
\text { only } \\
n\end{array}$ \\
\hline Sarcoidosis & 17 & $5 / 12$ & 4 & 2 & & \\
\hline FA CFA & 10 & $9 / 1$ & 7 & 1 & 4 & \\
\hline Rheumatic* & 4 & $1 / 3$ & 4 & 2 & 1 & \\
\hline $\begin{array}{l}\text { Systemic } \\
\text { sclerosis }\end{array}$ & 20 & $9 / 11$ & 12 & 3 & 2 & 4 \\
\hline Other ILD & 3 & & & & & \\
\hline
\end{tabular}

M: male; F: female; Pred: prednisolone; Im: immunosuppression; FA: fibrosing alveolitis; CFA: cryptogenic fibrosing alveolitis; ILD: interstitial lung disease. *: rheumatic disease other than system sclerosis; +: including exsmokers. tures combined with physiological and radiological (including thin section computed tomography (CT)) assessment and confirmed by biopsies in 30 patients (13 sarcoidosis, three other, seven FA (5 CFA), and seven with FA and SSc (FASSc)). As a control group, 16 healthy normal individuals underwent BAL as controls (10 males and six females, aged 18-52 yrs, no current or exsmokers; all receiving no treatment). The approval of the Royal Brompton National Heart and Lung Hospital Ethics Committee was obtained for the study of normal subjects.

Bronchoalveolar lavage. BAL was carried out according to standard unit protocol. In brief, following premedication with atropine and papavaretum, routine fibreoptic bronchoscopy was performed. Lavage was undertaken in three regions of the lungs (the medial segment, right middle lobe; lateral basal segment, right lower lobe; and superior segment, lingula) by instilling $100 \mathrm{~mL}$ warmed saline in $5 \times 20 \mathrm{~mL}$ aliquots with gentle suction after each aliquot. Samples were collected on ice before immediate transportation to the laboratory where the cells were counted, assessed for viability by trypan blue exclusion and pelleted by centrifugation at $700 \times \mathrm{g}$ for $10 \mathrm{~min}$. The supernatant fluid was then removed, aliquoted and stored at $-70^{\circ} \mathrm{C}$ until further analysis. The cell pellet was resuspended in RPMI 1640 medium (Chester Beatty Laboratories, London, UK) at $2 \times 10^{5}$ cells $\cdot \mathrm{mL}^{-1}$ and cytocentrifuge preparations made using a Shandon II cytocentrifuge (Shandon Southern Instruments, Sewickly, PA, USA). Following staining with May-Grunwald Giemsa, differential cell counts were performed by counting a minimum of 200 cells. Abnormal elevations of BAL differential cell counts were defined using published normal ranges for BAL counts [12]: neutrophils normal range $<3 \%$; eosinophils normal range $<0.5 \%$ and lymphocytes normal range $<15 \%$.

Measurement of BAL albumin. The concentration of albumin in BAL fluid was measured using a colorimetric assay utilizing specific binding of albumin with bromocresol green. We used a commercially available kit for the measurement of serum albumin (Sigma Chemical Co. Ltd., Poole, UK), which was modified in order to estimate BAL fluid albumin levels as previously reported [13]. In brief, $1 \mathrm{~mL}$ aliquots of BAL fluid were combined with an equal volume of bromocresol green and the reaction allowed to proceed at room temperature for $10 \mathrm{~min}$. A standard curve was generated by treating serial dilutions of a standard solution of albumin in an identical manner. The absor-bance of each sample was measured at $698 \mathrm{~nm}$ on a Gilford 2600 spectrophotometer (Corning Ltd, Halstead, UK).

Evaluation of bronchoalveolar lavage fluid for soluble adhesion cell molecules. Two-site enzyme-linked immunoassays (ELISAs) were constructed from pairs of monoclonal antibodies for ICAM-1 (CD54), VCAM-1 (CD106) and E-selectin (CD62E). The assays were calibrated with standard preparations of recombinant soluble forms of ICAM-1, VCAM-1 and E-selectin, which were assigned arbitrary units ( 1 unit ICAM-1 = $4.5 \mathrm{ng}$; 1 unit VCAM- $1=$ $9 \mathrm{ng} ; 1$ unit E-selectin = $2 \mathrm{ng}$ ). The detection limits of each assay were: ICAM-1 2.0 U.mL-1; VCAM-1 $1.5 \mathrm{U}$. $\mathrm{mL}^{-1}$; and E-selectin $0.2 \mathrm{U} \cdot \mathrm{mL}^{-1}$ [14]. 
The ELISA methodology has been reported previously [15]. In brief, microwell plates (Immulon, Nunc Inc, Life Technologies Ltd, Paisley, UK) were precoated with 100 $\mu \mathrm{L}$ of a $10 \mu \mathrm{g} \cdot \mathrm{mL}^{-1}$ solution of specific antibody in $0.1 \mathrm{M}$ carbonate butter (pH 8.9). The antibodies used were: antiICAM-1 (BBIG-11), anti-VCAM-1 (BBIG-V4), and antiE-selectin (BBIG-E1). One hundred microlitres of BAL fluid was added to the wells and incubated for $2 \mathrm{~h}$ at room temperature. After washing, any bound adhesins were detected by the addition of $100 \mu \mathrm{L}$ of biotinylated sec- ond antibody $\left(1 \mu \mathrm{g} \cdot \mathrm{mL}^{-1}\right)$, followed by $100 \mu \mathrm{L}$ of a 1:000 dilution of streptavidin-horse radish peroxidase (HRP) (Amersham International plc, Little Chalfont, UK). The biotinylated antibodies used were: anti-ICAM-1 (BBIG12); anti-VCAM-1 (BBIG-V3); anti-E-selectin (BBIG-E5). Bound HRP was detected by the addition of $50 \mu \mathrm{L}$ of the substrate tetramethylenzidine (Universal Biologicals, Kingston Upon Thames, UK). The reaction was incubated for $10 \mathrm{~min}$ then stopped by the addition of $0.2 \mathrm{M}$ citric acid. The colorimetric reaction product was measured at $405 \mathrm{~nm}$ on a Titertek Multiscan plate reader (Flow Labs, Rickmansworth, UK).

\section{Immunohistology}

Subjects. The patient group comprised 11 patients with FA occurring in the context of SSc (four males and seven females; ages 32-66 yrs; seven current or exsmokers, two receiving treatment with prednisolone both of whom were also receiving immunosuppression, and eight receiving no treatment) and four with CFA (two males and two females, ages 42-57 yrs, three current or exsmokers, all four receiving no treatment) who underwent open lung biopsy at presentation for confirmation of diagnosis and staging of activity of the interstitial process. As disease controls, peripheral uninvolved lung was obtained from resection specimens from 11 patients undergoing thoracotomy (eight non-small cell carcinoma of the lung, one secondary histiocytoma, one carcinoid tumour, one scar with dystrophic calcification, seven males and four females, ages 45-79 yrs, seven current or exsmokers, all receiving no treatment).

Preparation of biopsy specimens. Lung biopsies were placed in optimum cutting temperature (OCT) medium (Gurr, BDH Limited, Poole, UK) on small cork discs and frozen in isopentane cooled in a bath of liquid nitrogen. The snap-frozen tissues were stored at $-20^{\circ} \mathrm{C}$ until further use. Sections $(8 \mu \mathrm{m})$ were cut on a cryostat and transferred to poly-L-lysine coated slides. These were air dried, fixed in a mixture of chloroform and acetone (1:1), wrap-ped in cling film and stored at $-20^{\circ} \mathrm{C}$ until use.

Histology. Sections from all biopsy specimens were stained with haematoxylin and eosin to examine morphology.

Immunohistology. Lung biopsies were examined by immunohistology for ICAM-1 (CD54), VCAM-1 (CD106) and E-selectin (CD62E). In addition we examined PECAM -1 (CD31), an adhesion molecule constitutively expressed by endothelial cells, to enable us to relate the expression of ICAM-1, VCAM and E-selectin to the amount of vascular endothelium present in the lung biopsies [16].
Alkaline phosphatase anti-alkaline phosphatase (APAAP) methodology was used to identity adhesion molecules in the tissues using Fast Blue (Sigma) as the chromogen. Mouse monoclonal antibodies directed against human ICAM-1, VCAM-1, E-Selectin and PECAM were a generous gift of British Bio-technology (Oxford, UK). Sections were examined by light microscopy for fast blue positivity. As a preliminary measure, we performed a semiquantitative analysis of ICAM-1 expression in all subjects and descriptive assessment of E-selectin, VCAM-1 and PECAM in a subgroup of FASSc and control subjects. ICAM-1 positive staining was graded according to the percentage of alveolar epithelium stained $(0-25 \%, 25-50 \%, 50-75 \%,>75 \%)$ and all intact alveoli were counted in triplicate for presence/absence of ICAM-1 positivity. Having confirmed the suitability of the methodology, the expression of the four adhesion molecules was quantified by point counting, using biopsy material from four CFA, two FASSc and four controls. Lung biopsies were stained as above, and were assessed by point counting using a 100-point graticule (Graticules Limited, Tonbridge, UK). Whenever a point overlaid tissue, it was scored for pre-sence or absence of test blue positivity. All available consecutive fields were counted. Analysis of counts of cumulative fields showed that variation was minimized after counting 30 fields. The count obtained from 30 fields per adhesion molecule per subject was subsequently used for analysis. The data were analysed for adhesion molecule positivity, both absolute and related to PECAM-1 expression.

\section{Analysis of adhesion molecule gene expression}

Ribosomal nucleic acid (RNA) extraction. Lung biopsy samples were obtained from five patients with FASSc who underwent open lung biopsy for confirmation of the diagnosis and staging of inflammatory activity. As controls, a small piece of peripheral uninvolved lung was obtained from five subjects undergoing thoracotomy for resection of tumour (four non small cell carcinoma of lung, one leiomyosarcoma of oesophagus). Lung tissue was stored under liquid nitrogen until RNA extraction.

The lung biopsy material was homogenized using an Ultra-turrax homogenizer (Janke and Kunkel, Breisgau, Germany) in $4 \mathrm{M}$ guanidium isothiocyanate denaturing solution and stored at $-80^{\circ} \mathrm{C}$ until RNA extraction. Total cellular RNA was extracted using the single step acid guanidium thiocyanate phenol chloroform method [17]. The RNA pellets were redissolved in water and stored at $-80^{\circ} \mathrm{C}$ after the addition of $1 \mathrm{~mL}$ of ribonuclease (RNAse) inhibitor (Promega Corporation, Southampton, UK).

As a preliminary assessment, $1 \mathrm{mg}$ of lung biopsy RNA was subjected to RT-PCR amplification for the constitutively expressed $\beta$-actin, to check the adequacy of the extracted RNA and the reverse transcription process. Amplification using primers for the histidyl transfer RNA (tRNA) synthetase gene [18] were used to screen for contamination of the RNA by genomic DNA.

Adhesion molecule gene expression. Gene expression of E-selectin, VCAM-1 and ICAM-1 was assessed using RTPCR methodology using primers that have previously been described [19]. In brief, $0.4 \mathrm{~mL}$ aliquots of the RNA samples were reverse transcribed using AMV reverse 
transcriptase (Northumbria Biologicals Limited, UK) and then underwent 30 cycle polymerase chain reaction (PCR) in the same reaction tube. The expected sizes of the PCR am-plification products were, thus, E-selectin 236 base pairs, ICAM-1 221 base pairs and VCAM-1 241 base pairs. The PCR products were visualized using $2 \%$ agarose gel electrophoresis and ethidium fluorescence.

\section{Statistical analysis}

The three subjects who had SSc without evidence of FA on high resolution computed tomography scan (HRCT) scan were excluded from statistical analysis as they did not clearly belong to either "Lung disease present" or "Normal" groups.

Comparisons of groups were made using the MannWhitney $U$ test and the Fisher exact probability test. Correlations were measured using Spearman's rank correlation coefficient, a nonparametric measure of correlation. Because of the size of the groups, data were assumed to be non-normally distributed and are expressed as median and range for each group.

To test the assumption that the groups were not normally distributed, comparisons between groups were also made on both raw and log-transformed data using Student's t-tests and one-way analysis of variance (ANOVA).

\section{Results}

Soluble adhesion molecules in bronchoalveolar lavage fluid

To determine whether soluble adhesion molecules were present in BAL fluid we used two-site immunoassays to measure adhesion molecule expression.

ICAM-1 (CD54). All but two subjects (one FASSc, one sarcoidosis) had detectable soluble ICAM-1 in BAL fluid. There was no difference in the amount of soluble ICAM-1 between subjects with lung disease and normal subjects

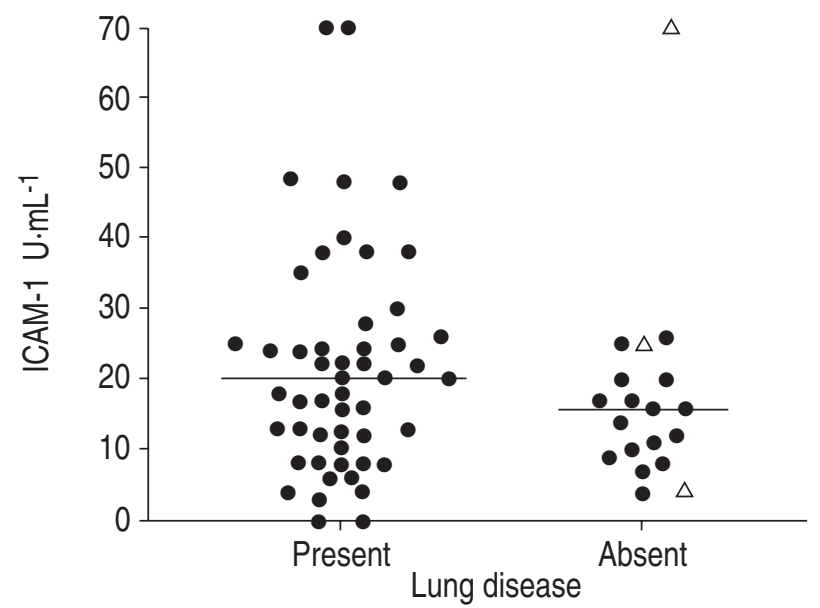

Fig. 1. - Soluble intercellular adhesion molecule-1 (ICAM-1) (CD54) in bronchoalveolar lavage fluid (all subjects). Each point represents the soluble ICAM-1 concentration in bronchoalveolar lavage (BAL) fluid from one subject. $\Delta$ : patients with systemic sclerosis who had no evidence of fibrosing alveolitis. Horizontal lines represent the median for each group. There was no difference between subjects according to the presence or absence of lung disease. (fig. 1). Furthermore, ICAM-1 concentration was not different between the separate disease entities, either as absolute values or as a ratio of soluble ICAM-1 to albumin to control for variations in endothelial permeability (fig. 2). The median (range) absolute concentration of soluble ICAM-1 in normal subjects was 15 (4-26) U.mL-1, in FA (including subjects with CFA and four with FA in the context of other rheumatological disorders) $20(8-40) \mathrm{U} \cdot \mathrm{mL}^{-1}$, in FASSc $8(0-70) \mathrm{U} \cdot \mathrm{mL}^{-1}$ and in sarcoidosis $24(0-48)$ $\mathrm{U} \cdot \mathrm{mL}^{-1}$. The levels in the six subjects not included in the statistical analysis of disease groups were: 4, 20 and 75 $\mathrm{U} \cdot \mathrm{mL}^{-1}$ in SSc without $\mathrm{FA}$; and 13,17 and $22 \mathrm{U} \cdot \mathrm{mL}^{-1}$ in other ILD.

E-selectin $(C D 62 E)$. In contrast to ICAM-1, E-selectin was only detected in 17 out of 70 individuals (one normal, two FA (one CFA and one rheumatoid arthritis with FA), five FASSc, seven sarcoidosis and two other ILD (one hypersensitivity pneumonitis and one asbestos-related ILD)). The presence of soluble E-selectin was related to the presence of interstitial lung disease (Fisher's exact probability, $\mathrm{p}=0.0173$ ) (fig. 3). To investigate whether the presence of soluble E-selectin was related to an abnormal accumula-
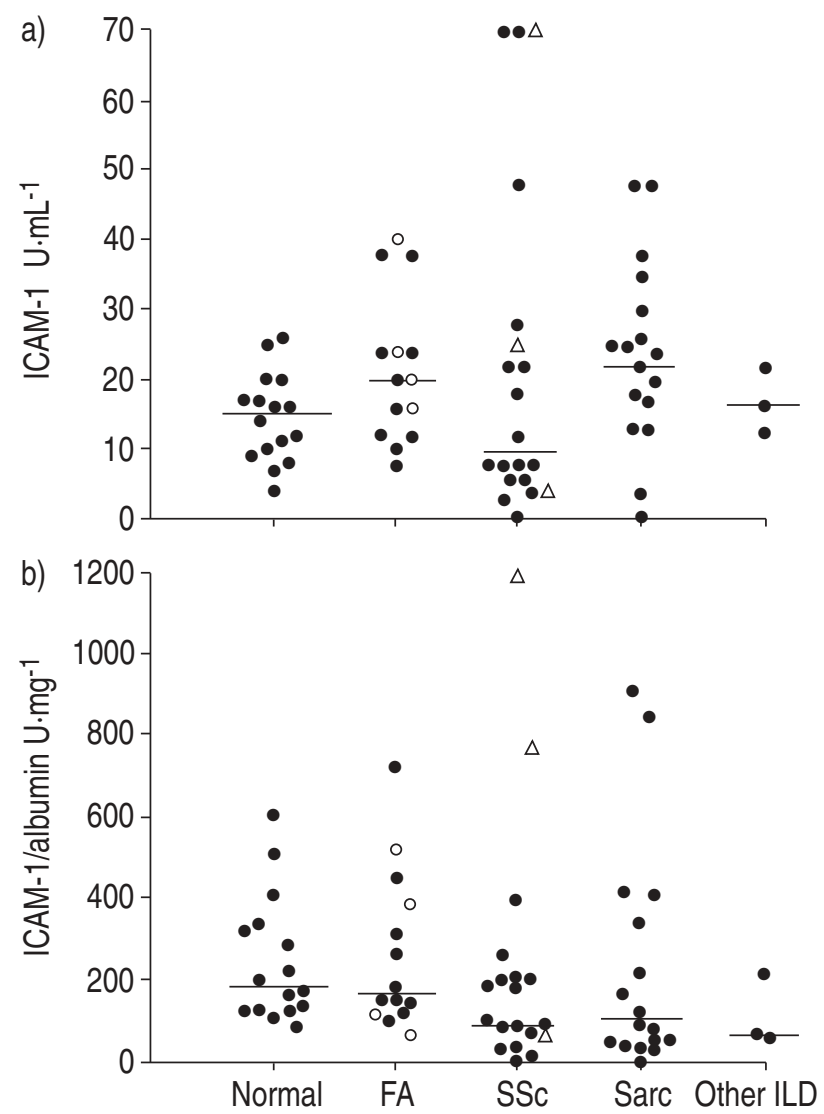

Fig. 2. - Soluble intercellular adhesion molecule-1 (ICAM-1) (CD54) in bronchoalveolar lavage fluid according to disease entities: a) absolute concentration of ICAM-1 in bronchoalveolar lavage (BAL) fluid; and b) ICAM-1 concentration corrected for albumin concentration. Each point represents one subject. $\bigcirc$ : patients with fibrosing alveolitis (FA) occurring in the context of a rheumatic disease other than systemic sclerosis (SSc); $\Delta$ : patients with SSc who had no evidence of FA. These patients were excluded from the statistical analysis, as was the group of three patients with other interstitial lung diseases (ILD). Horizontal lines represent the median for each group. There was no statistical difference in ICAM-1 concentration between the groups. Sarc: sarcoidosis. 


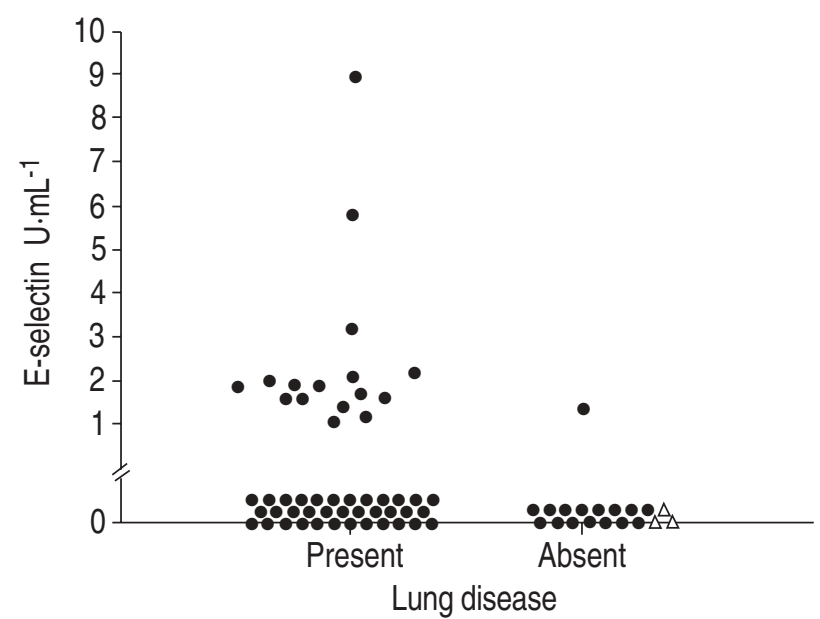

Fig. 3. - Soluble E-selectin (CD62E) in bronchoalveolar lavage fluid (all subjects). Each point represents one subject. $\Delta$ : patients with systemic sclerosis who had no evidence of fibrosing alveolitis. The association between detectable E-selectin and the presence of interstitial lung disease was statistically significant $(\mathrm{p}=0.0173)$.

Table 2. - Relationship between soluble E-selectin and lymphocytosis in bronchoalveolar lavage (BAL) fluid

\begin{tabular}{lcc}
\hline $\begin{array}{l}\text { Lymphocytes } \\
\text { in BAL }\end{array}$ & $\begin{array}{c}\text { E-selectin } \\
\text { present }\end{array}$ & $\begin{array}{c}\text { E-selectin } \\
\text { absent }\end{array}$ \\
\hline Elevated (Š15\%) n & 11 & 14 \\
Normal $(<15 \%) \mathrm{n}$ & 6 & 36
\end{tabular}

The presence of soluble E-selectin in BAL fluid, as determined by two-site enzyme-linked immunosorbent assay (ELISA) in all subjects where BAL differential cell count was available, was strongly associated with an increased percentage of lymphocytes in BAL fluid determined by cell counts on cytocentrifuge preparations of BAL fluid ( $<<0.01$; Fisher's exact test).

tion of inflammatory cells within the lower respiratory tract we examined the relationship between E-selectin expression and abnormal elevation of differential counts of BAL cell populations. Detectable soluble E-selectin was significantly associated with the presence of a BAL lymphocytosis (Fisher's exact probability $\mathrm{p}=0.0069$ ) (table 2 ). There was no association between the presence of soluble E-selectin in BAL fluid and either a BAL neutrophilia or eosinophilia. In patients with detectable lung disease, BAL albumin concentration was significantly higher in subjects with detectable E-selectin (Mann-Whitney U, p< $0.05)$.

VCAM-1 (CD110). Soluble VCAM-1 was rarely detected in BAL fluid, being present in two of the 16 normal subjects, one of the 14 with FA, none of those with SSc, two of the 17 with sarcoidosis and none of those with other ILD.

Immunohistological evaluation of the expression of ICAM -1, VCAM-1, E-selectin, and PECAM-1

The expression of ICAM-1, VCAM-1, E-selectin and PECAM-1 was evaluated semiquantitatively and quantitatively.

Semiquantification. In our preliminary studies, lung material from 11 FASSc, four CFA, and 11 control subjects were examined for surface expression of ICAM-1. ICAM -1 was present in extensive areas of biopsies obtained from patients with FA and controls, and was particularly prominent on the alveolar surface epithelium (fig. 4). Scattered intraluminal cells also demonstrated immunoreactivity for ICAM-1.

All alveoli were assessed for the presence/absence of ICAM-1. In FA (CFA and FASSc), $96.1 \pm 5.8 \%$ of all alveoli were positive for ICAM-1 compared with $99.7 \pm 0.9 \%$ of alveoli from control subjects.

To estimate the extent of ICAM-1 expression, the proportion of the length of the alveolar surface epithelium was assessed semiquantitatively; 13 of the $15 \mathrm{FA}$ and all of the 11 controls showed ICAM-1 positivity along $>75 \%$ of the length of the alveolar epithelial surface. There was no statistical difference in ICAM-1 staining between the lung of FA patients and that of control subjects.

In our screening studies of VCAM-1, E-selectin and PECAM we assessed the degree and distribution of immunoreactivity for these adhesion molecules. VCAM-1 positivity was occasionally seen in a patchy distribution around

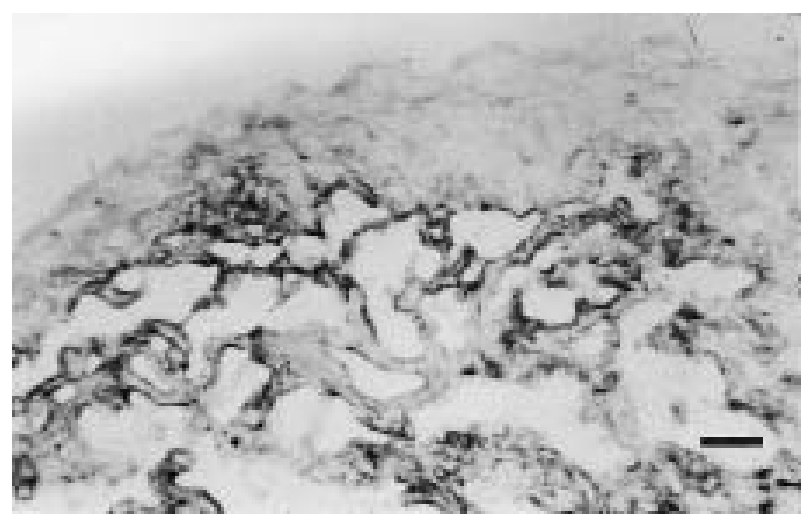

Fig. 4. - Immunohistology of ICAM-1 (CD54) expression within the lung parenchyma. The micrograph is an example from a patient with fibrosing alveolitis in association with systemic sclerosis. Intercellular adhesion molecule-1 (ICAM-1) expression was detected using alkaline phosphatase anti-alkaline phosphatase methodology with fast blue as the substrate. ICAM-1 staining was intense and was expressed along virtually the entire alveolar surface epithelium. Internal scale bar $=25 \mu \mathrm{m}$.

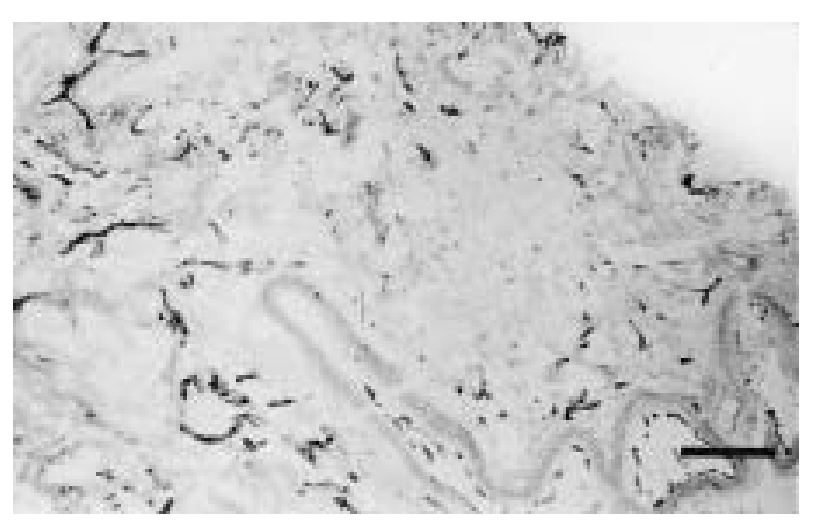

Fig. 5. - Immunohistology of E-selectin (CD62E) expression within the lung parenchyma. The micrograph is an example from a patient with fibrosing alveolitis in association with systemic sclerosis. E-selectin expression was detected using alkaline phosphatase anti-alkaline phosphatase methodology with fast blue as the substrate. The distribution of E-selectin positivity was patchy, and was found around moderate and large blood vessels. Internal scale bar $=100 \mu \mathrm{m}$ 
Table 3. - Adhesion molecule immunoreactivity in open lung biopsies: point counting

\begin{tabular}{lccc}
\hline & Control & FA & p-value \\
\hline ICAM-1 \% & 85.45 & 89.55 & 0.09 \\
& $(21.3)$ & $(11.3)$ & \\
VCAM \% & 9.5 & 10.55 & 0.18 \\
& $(14.0)$ & $(21.8)$ & \\
E-selectin \% & 9.4 & 9.1 & 0.55 \\
& $(14.0)$ & $(7.9)$ & \\
PECAM \% & 76.3 & 86.9 & 0.46 \\
& $(33.8)$ & $(17.4)$ & \\
ICAM-1/PECAM & 1.16 & 1.11 & 0.55 \\
VCAM/PECAM & $(1.00)$ & $(0.27)$ & 0.38 \\
E-Selectin/PECAM & 0.14 & 0.11 & $0.38)$ \\
& $(0.23)$ & $(0.38)$ & 0.45 \\
\hline
\end{tabular}

$\overline{\text { Frequency of adhesion molecule immunoreactivity was deter- }}$ mined by point counting. The data are shown as median (range) of percentage of positive points over total number of points overlying lung parenchyma in peripheral uninvolved lung from four subjects undergoing tumour resection (control) and open lung biopsy samples from six patients with fibrosing alveolitis (FA) (four cryptogenic FA and two associated with systemic sclerosis). The data are presented both as absolute results and related to platelet/endothelial cell adhesion molecule (PECAM) staining of endothelium. Mann Whitney U-tests were used in the statistical analysis.

a)

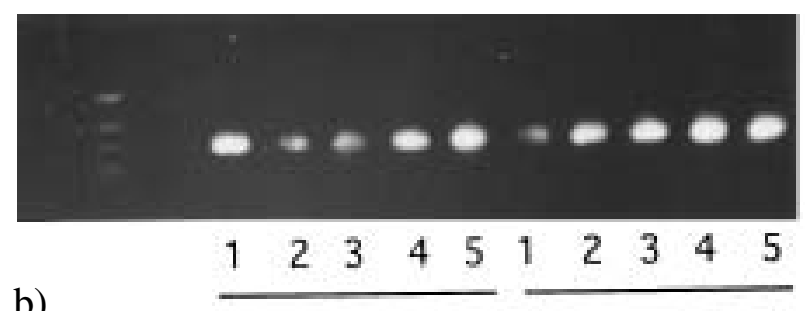

b)

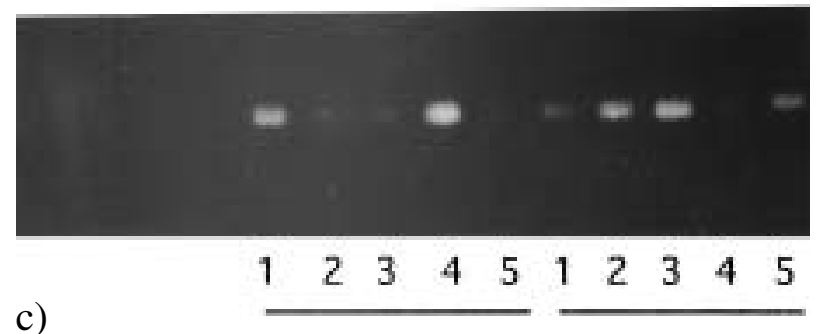

c)

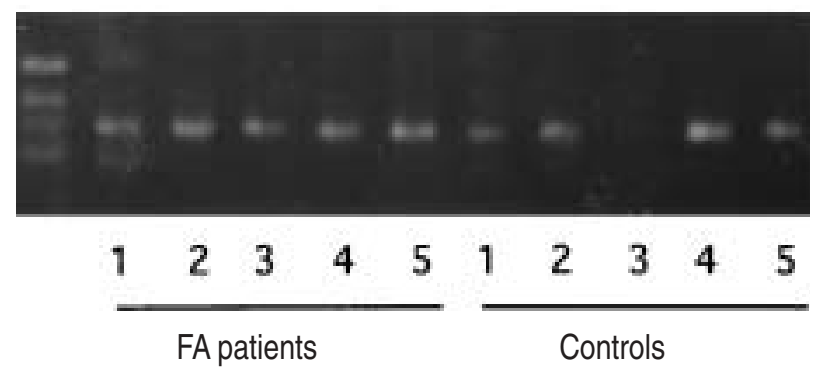

Fig. 6. - Expression of messenger ribonucleic acid (mRNA) encoding E-selectin (CD62E); intercellular cell adhesion molecule-1 (ICAM-1) (CD54) and vascular cell adhesion molecule-1 (VCAM-1) (CD110) in lung tissue: examples of amplification products for the genes encoding a) E-selectin, b) ICAM-1 and c) VCAM-1 in lung biopsy material from each of five patients with fibrosing alveolitis (FA) and five control subjects using ribonucleic acid extraction/reverse transcription-polymerase chain reaction. blood vessels. E-selectin staining was patchy, and was found related to moderate-to-large blood vessels. Rarely, some E-selectin positivity was observed in the interstitium in FASSc subjects (fig. 5). PECAM-1 staining was widespread in both FASSc and controls and was predominantly interstitial, with sparing of the alveolar epithelial surface.

Quantification. A quantitative assessment of adhesion molecule immunoreactivity was performed by point counting of lung parenchyma from six FA patients and four controls using monoclonal antibodies to ICAM-1, VCAM -1 , E-selectin, and PECAM. The results are shown in table 3: there were no differences in the amount of adhesion molecule staining between patients and controls for any of the four adhesion molecules used. When the results were related to PECAM positivity, the lack of statistical difference between patients and controls persisted.

\section{Adhesion molecule gene expression in open lung biopsy specimens using RT-PCR}

Expression of mRNA coding for E-selectin, ICAM-1 and VCAM-1 was detectable by RT-PCR in lung tissue derived from each of five patients with FA and also from five controls (fig. 6). No attempt was made to quantify mRNA expression with this technique.

\section{Discussion}

This study has shown that soluble adhesion molecules, cell surface-related adhesion molecules and mRNA for ICAM-1 (CD54), VCAM-1 (CD110) and E-selectin (CD$62 \mathrm{E}$ ) can be identified within the lungs of patients with diffuse lung disease. We found that soluble E-selectin in BAL fluid was the only adhesion molecule present in greater amounts in disease than controls. Strikingly, with this one exception, no significant differences between diseased lung and controls were found for any of the indices of adhesion molecule expression, suggesting that adhesion molecules play important roles in the normal function of this immunologically active organ. This is consistent with the observation that the normal lung contains large numbers of T-cells that carry the phenotype of the "memory" subset of cells [20] which are capable of an enhanced response to recall antigens (CD45RO+), and is likely to be important for immune surveillance.

\section{Adhesion molecule expression and lung inflammation}

In order for inflammatory cells such as neutrophils, eosinophils, lymphocytes and monocytes to migrate from the vascular compartment into disease sites, these cells must first adhere to the endothelial surface prior to their migration into the interstitium. Upregulation of adhesion molecule expression has been postulated as a factor in the persistence of inflammation $[4,5]$.

Our studies have confirmed the presence at both the transcription and translational level of E-selectin, ICAM-1 and VCAM-1 within the lungs of patients with fibrosing 
lung disease and also in normal controls. Furthermore, we have been unable to establish any quantitative difference in expression of these molecules between normal and disease. This suggests that the normal lung is actively involved in trafficking of inflammatory cells into the interstitium. As this does not result in pulmonary disease it is likely that these mechanisms are part of normal lung homeostasis.

The results of this study did not demonstrate increased expression of adhesion molecules in lung fibrosis and there are two possible explanations for this. Firstly, it was not possible to quantify the density of adhesion molecule expression using immunohistology. It is, therefore, possible that, despite the similar distribution of adhesion molecule expression in normal and diseased states, that at the cellular level there were major differences in the numbers of molecules expressed at the cell surface. Secondly, it is possible that the source of control material, lung peripheral to a central tumour, was not truly normal. This cannot be excluded unequivocally. However, the lung in this region was normal to light microscopy and well distant from the proximal malignancy. It is unlikely that local responses to the tumour would have had such a widespread effect on the remaining lung.

It is also possible that it is expression of the ligands for the adhesion molecules on the surface of the immune effector cells which are the prime determinants of traffic to disease sites. In this regard, inflammatory cell ligands such as the leucocyte adhesion molecules $\left(\beta_{2}\right.$ integrin CD18 combined with one of three different chains (leucocyte function associated antigen (LFA-1) (CD11a); Mac-1 (CD11b); and p150,95 (CD11c))) and IGSF member macrophage ICAM-1 (CD54) have been shown to be upregulated in diffuse inflammatory lung diseases [21, 22]. Therefore, the relative contributions of adhesion molecule expression and inflammatory cell ligand expression will require ultrastructural studies to provide a more detailed elucidation.

\section{Distribution of adhesion molecule expression in the lungs}

Although the expression of E-selectin and VCAM-1 was, as predicted, restricted to the endothelial surfaces, the striking feature was the very widespread expression of ICAM-1 along the alveolar epithelium. This observation is similar to the findings of MONTEFORT et al. [10] of ICAM-1 expression along the length of the bronchial epithelium in normal and asthmatic subjects. Widespread epithelial expression of ICAM-1 may be involved in the pathogenesis of epithelial cell damage which is widely regarded as one of the first abnormalities to occur in the pathogenesis of fibrosing alveolitis [23].

\section{The significance of soluble adhesion molecules in lung lavage fluid}

The most striking observation in our studies has been the finding of large amounts of ICAM-1 and, in selected individuals, E-selectin in bronchoalveolar lavage samples. ICAM-1 was found universally and was detected in equivalent amounts in normal subjects and in patients with a variety of pulmonary diseases characterized by chronic interstitial inflammation. This universal presence is con- sistent with the immunohistochemical findings of widespread ICAM-1 expression on the alveolar surfaces in both disease and control groups. The significance of this shedding into the epithelial lining fluid is not clear but may well represent a control mechanism to prevent a persistent and prolonged adhesion of inflammatory cells to the epithelial cells.

In striking contrast to this universal expression of ICAM-1, soluble E-selectin was detected almost exclusively in patients with pulmonary disease and was strongly correlated with the finding of excess lymphocyte numbers on the epithelial lining surface. The expression of E-selectin on high endothelial venules has been proposed as an important factor in the development of vascular leak [24, 25]. Consistent with this, albumin levels in bronchoalveolar lavage fluid from pulmonary disease were significantly higher in samples that were positive for E-selectin when compared with disease subjects without detectable E-selectin. Increased vascular permeability is a rational explanation for our finding of an adhesion molecule that is known to be expressed almost exclusively on endothelial cells. Its absence from normal lung lavage fluids would be consistent with the absence of vascular leak and would also explain why no soluble molecules were observed despite their presence on normal endothelial cells examined by immunohistology.

The restriction of soluble E-selectin to disease states suggests that soluble E-selectin may be useful as a marker of disease activity, but this needs to be explored further.

The association between E-selectin and a raised percentage of lymphocytes on the epithelial lining surface is intriguing. The ligand for E-selectin is sialylated Lewis $\mathrm{x}$ (CD15s) and related structures [26] which is found in abundance on neutrophils and monocytes. E-selectin also mediates adherence to a subpopulation of "memory" T-lymphocytes bearing the cutaneous lymphocyte antigen that appear to be selectively recruited to inflammatory/ immune reactions in the skin [27]. It is possible to speculate that E-selectin mediates the recruitment of a similar subset of T-cells to disease sites in lung disease.

Our studies found no relationship between the expression of E-selectin and neutrophil influx in contrast to studies of MONTEFORT et al. [28] examining patients with perennial allergic rhinitis in whom increased expression of E-selectin was found in the patient group. The functio-nal roles of adhesion molecules at disease sites still need to be elucidated, but at present it would appear that the major value of identifying a presence of soluble adhesion molecules such as E-selectin and VCAM-1 lies in their use as markers of inflammation and they may indeed provide more sophisticated indices of change in inflammatory state in response to therapy. These roles will need to be defined with more longitudinal studies.

In conclusion, we have demonstrated that the adhesion molecules intercellular adhesion molecule-1, E-selectin and vascular cell adhesion molecule- 1 are present in normal lung and in patients with fibrosing alveolitis. We have been unable to identify differential expression of these adhesion molecules, which would have suggested that their regulation played a key role in the pathogenesis of fibrosing lung disease, but rather suggested that they may play a role in normal lung homeostasis. The association of the soluble adhesion molecule E-selectin with a lymphocytic alveolitis requires further study. 


\section{References}

1. Crystal RG, Bitterman PB, Rennard ST, Hance AJ, Keogh BA. Interstitial lung diseases of unknown cause: disorders characterized by chronic inflammation of the lower respiratory tract. $N$ Engl J Med 1984; 310: 154-165 and 235-244.

2. Albelda SM, Buck CA. Integrins and other cell adhesion molecules. FASEB J 1990; 4: 2868-2880.

3. Butcher EC. Leukocyte-endothelial cell recognition: three (or more) steps to specificity and diversity. Cell 1991; 67: 1033-1036.

4. Koch AK, Burrows JC, Haines GK, et al. Immunolocalization of endothelial and leukocyte adhesion molecules in human rheumatoid and osteoarthritic synovial tissues. Lab Invest 1991; 64: 313-320.

5. Pitzalis C, Kingsley G, Panayi G. Adhesion molecules in rheumatoid arthritis: role in the pathogenesis and prospects for therapy. Ann Rheum Dis 1994; 53: 287-288.

6. Rothlein R, Mainolfi EA, Czajkowski M, Marlin SD. A form of circulating ICAM-1 in human serum. $J$ Immunol 1991; 147: 3788-3793.

7. Seth R, Raymond FD, Makgoba MW. Circulating ICAM-1 isoforms: diagnostic prospects for inflammatory and immune disorders. Lancet 1991; 338: 83-84.

8. Wellicombe SM, Kapahi P, Mason JC, Lebranchu Y, Yarwood H, Haskard DO. Detection of a circulating form of vascular cell adhesion molecule-1: raised levels in arthritis and systemic lupus erythematosus. Clin Exp Immunol 1993; 92: 412-418.

9. Wegner CD, Gundel RH, Reilly P, Haynes N, Letts LG, Rothlein R. Intercellular adhesion molecule-1 (ICAM- 1) in the pathogenesis of asthma. Science 1990; 247: 456459.

10. Montefort S, Roche WR, Howarth PH, et al. Intercellular adhesion molecule-1 (ICAM-1) and endothelial leucocyte adhesion molecule-1 (ELAM-1) expression in the bronchial mucosa of normal and asthmatic subjects. Eur Respir J 1992; 5: 815-823.

11. Shijubo N, Imai K, Aoki S, et al. Circulating intercellular adhesion molecule-1 (ICAM-1) antigen in sera of patients with idiopathic pulmonary fibrosis. Clin Exp Immunol 1992; 89: 58-69.

12. Technical recommendations and guidelines for bronchoalveolar lavage (BAL). Report of the European Society of Pneumology Task Group on BAL. Eur Respir J 1989; 2: 561-585.

13. Southcott AM, Jones KP, Li D, et al. Interleukin-8: differential expression in lone fibrosing alveolitis and systemic sclerosis. Am J Respir Crit Care Med 1995; 151: 16041612.

14. Gearing AJH, Hemingway I, Pigott R, Hughes J, Rees AJ, Cashman SJ. Soluble forms of vascular adhesion molecules, E-selectin, ICAM-1, and VCAM-1: pathological significance. Ann NY Acad Sci 1992; 667: 324-331.
15. Pigott R, Dillon LP, Hemingway IH, Gearing AJH. Soluble forms of E-selectin, ICAM-1 and VCAM-1 are present in the supernatants of cytokine activated cultured endothelial cells. Biochem Biophys Res Commun 1992; 187: 584-589.

16. Yan H-C, Juhasz I, Pilewski J, Murphy GF, Herlyn M, Albelda SM. Human/severe combined immunodeficient mouse chimeras. An experimental in vivo model system to study the regulation of human endothelial cell-leukocyte adhesion molecules. J Clin Invest 1993; 91: 986996.

17. Chomczynski P, Sacchi N. Single-step method of RNA isolation by acid guanidium thiocyanate-phenol-chloroform extraction. Anal Biochem 1987; 162: 156-159.

18. Corrochano LM. A test of human cDNA synthesis by the polymerase chain reaction. Genet Anal Tech Appl 1991; 8: 134-135.

19. Meagher L, Mahiouz D, Sugars K, et al. Measurement of mRNA for E-selectin, VCAM-1 and ICAM-1 by reverse transcription and the polymerase chain reaction. J Immunol Methods 1994; 175: 237-246.

20. Becker S, Harris DT, Koren HS. Characterization of normal human lung lymphocytes and interleukin- 2-induced lung T cell lines. Am J Respir Cell Mol Biol 1990; 3: 441-448.

21. Schaberg T, Rau M, Stephan H, Lode H. Increased number of alveolar macrophages expressing surface molecules of the CD11/CD18 family in sarcoidosis and idiopathic pulmonary fibrosis is related to the production of superoxide anions by these cells. Am Rev Respir Dis 1993; 147: 1507-1513.

22. Melis M, Gjomarkaj M, Pace E, Malizia G, Spatafora M. Increased expression of leukocyte function associated antigen-1 (LFA-1) and intercellular adhesion molecule-1 (ICAM-1) by alveolar macrophages of patients with pulmonary sarcoidosis. Chest 1991; 100: 910-916.

23. Harrison NK, Myers AR, Corrin B, et al. Structural features of interstitial lung disease in systemic sclerosis. Am Rev Respir Dis 1991; 144: 706-713.

24. Pober JS, Cotran RS. What can be learned from the expression of endothelial adhesion molecules in tissues? Lab Invest 1991; 64: 301-305.

25. Mulligan MS, Varani J, Dame MK, et al. Role of endothelial-leukocyte adhesion molecule 1 (ELAM-1) in neutrophil-mediated lung injury in rats. J Clin Invest 1991; 88: 1396-1406.

26. Phillips ML, Nudelman E, Gaeta FCA, et al. ELAM-1 mediates cell adhesion by recognition of a carbohydrate ligand, Sialyl-Lex. Science 1990; 250: 1130-1132.

27. Picker LJ, Kishimoto TK, Smith CW, Warnock RA, Butcher EC. ELAM-1 is an adhesion molecule for skinhoming T cells. Nature 1991; 349: 796-799.

28. Montefort S, Feather IH, Wilson SJ, et al. The expression of leukocyte-endothelial adhesion molecules is increased in perennial allergic rhinitis. Am J Respir Cell Mol Biol 1992; 7: 393-398. 